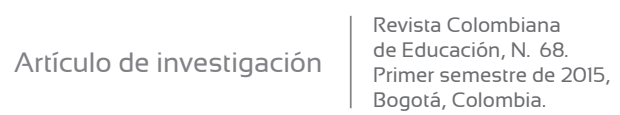

\section{Formar para investigar e innovar: tensiones y preguntas sobre la formación inicial de maestros en Colombia}

\author{
//Training for Research and Innovation \\ Tensions and Questions on Initial \\ Teacher Training in Colombia
}

//Formar para pesquisar e inovar:

tensões e perguntas sobre a formação inicial de docentes em Colômbia

\begin{abstract}
Fabiola Cabra Torres*
Dora Lilia Marín Díaz**

Recibido: 05/09/2014

Evaluado: $12 / 11 / 2014$

$16 / 11 / 2014$
\end{abstract}

\section{Resumen}

El artículo presenta algunos de los resultados del estudio titulado Formación en investigación e innovación en programas de licenciatura en los campos de Ciencias Sociales, Artes y Humanidades, Ciencias Naturales y Educación y Pedagogía, cofinanciado por el Ministerio de Educación y la Pontificia Universidad Javeriana ${ }^{1}$. En este se rastrearon y analizaron los objetivos y las estrategias de formación en investigación e innovación empleados por diferentes programas en los cuatro campos de conocimiento enunciados. Entre otros resultados, el estudio mostró que en las tres últimas décadas la investigación y la innovación fueron piezas claves de la tríada profesionalización, cualificación y actualización que orienta la preparación de los maestros, y que se inscribe en políticas de profesionalización docente que desplazan la atención de las prácticas de enseñanza propias de la acción de los maestros hacia tareas investigativas y de innovación destinadas a producir conocimiento pedagógico y transformaciones en el aula y la escuela.

\section{Abstract}

This article presents some of the results obtained in the study entitled Research and Innovation Training in Teacher Education Programs in Social Sciences, Arts and Humanities, Natural Sciences, Education and Pedagogy, financed jointly by the Ministry of Education of Colombia and the Pontificia Universidad Javeriana. The objectives and strategies used by programs to train future teachers in research and innovation were analyzed for the four fields mentioned. Among other findings, the study showed that, in the last decades, both innovation and research have been part of the triad teacher professionalization-qualificationupdating, which are key directions of the public policies driving teachers' education. Those guidelines move the focus on teaching towards researching and educational innovation activities, intended to produce pedagogical knowledge and transformation in the classroom and school.

1 Convenio 821 de 2012. En el análisis de datos participaron María Caridad García, Darcy Milena Barrios-Martínez, Jorge Andrés Jiménez Muñoz y Juan Carlos Garzón.

\section{Palabras clave}

Licenciatura, innovación, investigación, política educativa, formación inicial de maestros.

\section{Keywords}

Teacher Education Programs, innovation, research, educational policy, early teacher training.

\section{Palavras chave}

Licenciatura, inovação, pesquisa, política educativa, formação inicial de professores. 


\section{Resumo}

O artigo apresenta alguns dos resultados do estudo titulado Formação em pesquisa e inovação em programas de Licenciatura nos campos das Ciências Sociais, Artes e Humanidades, Ciências Naturais, e Educação e Pedagogia, co-financiado pelo Ministério da Educação e a Pontifícia Universidade Javeriana. Neste se rastrearam e analisaram os objetivos e as estratégias de formação em pesquisa e inovação, empregados pelos diferentes programas nos quatro campos de conhecimento enunciados. Entre outros resultados, o estudo sinalou que nas três ultimas décadas a pesquisa e a inovação foram peças essenciais da tríade profissionalização, qualificação e atualização que orienta a preparação dos professores, e que se inscreve em políticas de profissionalização docente, que movimentam a atenção das praticas de ensino próprias da ação docentes, em tarefas de pesquisa e inovação destinadas, a produzir conhecimento pedagógico e transformações na aula e na escola.

Como en otros países de América Latina, en Colombia la formación de los maestros es foco de atención no solo de un conjunto de políticas educativas expresadas en la legislación que sigue tendencias internacionales y que regula el sistema educativo nacional ${ }^{2}$, también lo es de las acciones que adelantan los movimientos de maestros así como otros intelectuales de la educación, quienes procuran reivindicar un lugar de saber para el maestro y, por eso mismo, el reconocimiento de saberes propios derivados de su práctica pedagógica que deberían orientar su formación inicial, profesional y permanente.

En las últimas dos décadas, las políticas de reforma educativa en los países de América Latina focalizaron su acción en el denominado factor docente (Vezud, 2011), como se reconoce en gran parte de las declaraciones internacionales, en las que el docente -el sujeto/objeto de las políticas públicas nacionales e internacionales- se presenta como protagonista y responsable del cambio educativo, como sujeto de los sistemas de evaluación y de control, y como receptor de capacitación permanente. Dicha focalización se ha caracterizado por el desplazamiento del problema de los maestros al "malestar docente" y la "dilución del maestro" por un énfasis en la "función docente", pero también por la centralidad del aprendizaje y la entrada en el

2 En este sentido el Estatuto Docente -Decreto 1278 de 2002, "por el cual se expide el estatuto de profesionalización docente"- estableció nuevas reglas de formación, ingreso, evaluación, promoción y permanencia en la profesión, las cuales buscan incidir en los resultados de los estudiantes mediante el denominado "mejoramiento de la calidad docente" 
escenario educativo de los entornos virtuales (Martínez Boom, 2000, 2004 y 2010a, 2010b).

Ese factor docente, del cual depende el cambio educativo y que requiere no solo de seguimiento, evaluación y control sino de capacitación permanente, aparece como una versión o posición que ocupan los maestros que actúan diariamente en las escuelas e instituciones educativas. De esta forma, la emergencia de la noción factor docente, así como su uso para responsabilizar a los maestros de los cambios que se supone necesitan los sistemas educativos de diversos países en América Latina, produjo un conjunto de discursos sobre la profesión docente caracterizados, entre otras cosas, por la reforma del trabajo que estos realizan (sus prácticas pedagógicas) y la formación de los maestros, que según Martínez Boom (2000) puede sintetizarse en un modo de profesionalización:

... que comprende los nuevos mecanismos para valorar, certificar y controlar los modelos institucionales de formación del profesorado y las prácticas pedagógicas en el aula. En consecuencia se trataría, según esta lógica de reinventar la profesión docente o, mejor, de convocar un proceso de "profesionalización" que tenga en principio dos ejes fundamentales:
1) que la transformación docente vaya articulada a la reconversión de la escuela y los sistemas educativos [...], y 2) que el nuevo diseño de la política parta de las necesidades y demandas de los usuarios y no de los intereses, problemas y necesidades de los maestros. La profesionalización descansa en la idea arraigada en ciertas corrientes de la investigación educativa que reclama para los maestros mayores competencias profesionales (pp. 98-99).

Ese núcleo discursivo de la profesionalización, en lo que se refiere a la formación de docentes, está soportado en diagnósticos que señalan, por ejemplo, el bajo nivel de formación que traen quienes ingresan a procesos de formación profesional; la poca calidad de los programas y los procesos de formación de los educadores; la falta de preparación de los agentes formadores; la tendencia a realizar una formación universalista, abstracta o poco diferenciada que no atiende las desigualdades sociales; la permanente tensión entre lógicas escolarizantes y lógicas académicas; la falta de regulación de los programas de formación ofrecidos; la escasa relación entre los contenidos de la formación y los espacios de desarrollo profesional, en particular la escuela; entre otros (Orealc/Unesco, 2012). 
A partir de estos diagnósticos, la profesionalización se dibuja como una categoría desde la cual es posible pensar la formación de los maestros hoy. Todo parece indicar que pensar la práctica del maestro en términos de profesión le ofrece a la formación otro contenido (Tenti, 2010): ella requiere la vinculación a instituciones especializadas que lo certifican como profesional y tiempos de preparación más o menos prolongados. Se espera que en esas instituciones y tiempos el maestro en formación acceda a contenidos y conocimientos que caracterizan su trabajo profesional y cuyo dominio le ofrezca autonomía frente al trabajo que debe realizar: "se le concede una notable capacidad de determinar las reglas que definen su trabajo y la evaluación de la calidad del mismo" (p. 43). Además la formación en la profesión supone que cada individuo va a consagrar la mayor parte de su tiempo a ejercer tal profesión y que de ella derivarán los recursos necesarios para su sustento económico y su posicionamiento social.

Un maestro más profesional es un maestro más cualificado, es decir, alguien que aplica conocimientos cada vez más complejos y formalizados y cuyo dominio efectivo requiere un esfuerzo cada vez más significativo (en cuanto a tiempo y recursos) (Tenti, 2010).

Sin embargo, es necesario reconocer que la profesionalización no supone el desarrollo de una capacidad profesional. Un tiempo de formación mayor no conduce obligatoriamente al "mejoramiento general de los conocimientos y de las aptitudes" y dicho mejoramiento no necesariamente hace "avanzar el proceso de profesionalización" (Hoyle, 1983, pp. 45-46 citado en Martínez Boom, 2010b, p. 28).

En términos generales, puede afirmarse que la profesionalización se orienta por criterios de competencia profesional y desempeño docente, gracias a los cuales "el maestro es incorporado a la política del 'conocimiento especializado' y del desarrollo tecnológico en general" (Martínez Boom, 2000, p. 100). En Colombia, en la década de 1990, la pregunta por la formación de los maestros (expresada ahora como formación docente) se vinculaba al desarrollo de competencias para la investigación y la innovación, dos temas acerca de los cuales hay cierto consenso y cuya importancia sería incuestionable.

Desde este momento, la discusión sobre la formación de los maestros se fundió a la formación, el desarrollo y la ampliación de las habilidades profesionales que no solo se garantizaban con la formación inicial, sino que debían orientar los procesos de cualificación docente, acciones dentro de las cuales la habilidad 
investigativa y la capacidad de innovar aparecen como condiciones imprescindibles. Se trató de un movimiento que acompañó la emergencia de cierta necesidad de formación permanente, una formación que cualifica la práctica pedagógica de los maestros, que desarrolla en ellos competencias profesionales y los hace responsables de su actualización permanente. Así, la tríada profesionalización, cualificación y actualización que emergió en la última década del siglo pasado, aparece como elemento orientador de las prácticas de formación docente dispuestas para la preparación de los maestros investigadores e innovadores en diferentes niveles (inicial, profesional, especializada, permanente, continuada, etc.).

La tabla 1 presenta el resultado del rastreo documental por medio del cual fue posible percibir la emergencia de estas nociones en las políticas que orientaron la formación de los maestros bajo la perspectiva de profesionalización. Allíse hace evidente el papel central que acciones asociadas a la investigación y la innovación alcanzaron en la definición de la formación profesional docente: una formación que deja de ocuparse de las prácticas de enseñanza y la ejercitación en el saber hacer y que comienza a instalarse en tareas investigativas destinadas a producir conocimiento pedagógico y con él transformaciones en el aula y la escuela. Así, investigación e innovación se presentan como objetivos de la formación de los maestros y tal como aparecen formulados en algunos documentos orientadores de política educativa, se constituyen en sus propósitos centrales.

Al respecto es necesario señalar que no dejan de aparecer reflexiones como las de Vaillant (2002), que destacan la necesidad de repensar esa orientación de la formación inicial de los profesores y volver la mirada a la necesidad de desarrollar cierta "competencia de clase o conocimiento del oficio" que le permita al maestro constituirse en un sujeto "diestro en la tarea de enseñar", hábil en su ejercicio práctico, lo que no significa que no pueda o deba tener una "actitud reflexiva acerca de su enseñanza" (p. 17). Según Galvin (2006), se trata de una formación del docente profesional que

$$
\begin{aligned}
& \text {... requiere el desarro- } \\
& \text { llo de programas que } \\
& \text { equilibren la teoría y la } \\
& \text { práctica, los contenidos } \\
& \text { científicos, los pedagó- } \\
& \text { gicos, los sociológicos, } \\
& \text { los psicológicos, a la par } \\
& \text { que los recursos metodo- } \\
& \text { lógicos para las distintas } \\
& \text { situaciones en el aula en } \\
& \text { contextos heterogéneos y } \\
& \text { multiculturales (p. 324). }
\end{aligned}
$$

Aun con reflexiones críticas y propuestas que llaman la atención sobre la necesidad de pensar la formación inicial de los maestros con relación a sus prácticas de enseñanza, se puede decir que, en general, durante las tres últimas décadas la investigación y la innovación se 
Tabla 1. La investigación y la innovación en planteamientos de la política educativa.

\begin{tabular}{|c|c|}
\hline $\begin{array}{l}\text { Documento } \\
\text { de política }\end{array}$ & $\begin{array}{l}\text { Objetivos relacionados con la investigación } \\
\text { e innovación educativa }\end{array}$ \\
\hline Ley 115 de 1994 & $\begin{array}{l}\text { Propone fortalecer la investigación en el campo pedagógi- } \\
\text { co. Es responsabilidad del Estado velar "por la cualificación } \\
\text { y formación de los educadores, la promoción docente, los } \\
\text { recursos y métodos educativos, la innovación e investiga- } \\
\text { ción educativa, la orientación educativa y profesional, la } \\
\text { inspección y evaluación del proceso educativo" (artículo } \\
\text { 4). }\end{array}$ \\
\hline $\begin{array}{l}\text { Decreto } 709 \text { de } \\
1996\end{array}$ & $\begin{array}{l}\text { Se establece que la formación inicial de docentes será im- } \\
\text { partida por las universidades, facultades de Educación y } \\
\text { escuelas normales (artículo 5). Se definen los campos de } \\
\text { formación docente para su mejoramiento profesional: pe- } \\
\text { dagógico, disciplinar, científico-investigativo, deontológi- } \\
\text { co, y en valores humanos (artículo 8). }\end{array}$ \\
\hline $\begin{array}{l}\text { Decreto } 272 \text { de } \\
1998\end{array}$ & $\begin{array}{l}\text { Exige a las facultades de Educación la existencia de por lo } \\
\text { menos una línea de investigación educativa para la acredi- } \\
\text { tación de los programas (artículo 6). }\end{array}$ \\
\hline $\begin{array}{l}\text { Decreto } 2566 \text { de } \\
2003\end{array}$ & $\begin{array}{l}\text { Establece la investigación como condición mínima para el } \\
\text { otorgamiento del registro calificado en todos los programas } \\
\text { profesionales, desarrollar la cultura investigativa y el pensa- } \\
\text { miento crítico en estudiantes y docentes (artículo 6). }\end{array}$ \\
\hline $\begin{array}{l}\text { Plan Decenal } \\
\text { de Desarrollo } \\
\text { Educativo } \\
1996-2005\end{array}$ & $\begin{array}{l}\text { Impulsa un programa de investigación: "Las universidades } \\
\text { y las Instituciones de ciencia y tecnología darán prioridad } \\
\text { a este programa. Con el fin de contar con un centro que } \\
\text { sirva de motor al proceso de innovación e investigación en } \\
\text { la educación, se creará en Colciencias una unidad especia- } \\
\text { lizada de investigación y aplicación pedagógica y didác- } \\
\text { tica" (cap. IV, segunda estrategia: Elevar la calidad de la } \\
\text { educación). }\end{array}$ \\
\hline $\begin{array}{l}\text { Plan Decenal de } \\
\text { Educación } \\
\text { 2006-2016 }\end{array}$ & $\begin{array}{l}\text { Propone "Crear concertadamente un sistema nacional de } \\
\text { formación, cualificación y promoción de estímulos para } \\
\text { docentes y directivos docentes, que articule los niveles de } \\
\text { formación inicial, pregrado y postgrado, cuyos ejes esencia- } \\
\text { les sean la investigación, la innovación, la pedagogía y la } \\
\text { didáctica" (Objetivo 4). "Fomentar, desarrollar y fortalecer, } \\
\text { de manera permanente, una cultura de ciencia, tecnología } \\
\text { e innovación" (Cap. Ciencia y tecnologías integradas a la } \\
\text { educación). }\end{array}$ \\
\hline $\begin{array}{l}\text { Resolución } 5443 \\
\text { de } 2010\end{array}$ & $\begin{array}{l}\text { Determina como requisito la investigación. Las institucio- } \\
\text { nes de educación superior formularán políticas de investi- } \\
\text { gación educativa, pedagógica y didáctica que fomenten la } \\
\text { reflexión, el pensamiento crítico, la indagación y el plan- } \\
\text { teamiento de soluciones innovadoras (artículo 7). }\end{array}$ \\
\hline $\begin{array}{l}\text { Decreto } 1295 \text { de } \\
2010\end{array}$ & $\begin{array}{l}\text { Para la adecuada formación se verificará que haya: "un am- } \\
\text { biente de investigación, innovación y creación, el cual exi- } \\
\text { ge políticas institucionales en la materia", una que incluya } \\
\text { estrategias para incorporar los resultados de la investigación } \\
\text { al quehacer investigativo y medios para la difusión de resul- } \\
\text { tados de investigación (artículo 5, numeral 5.5.3.1). }\end{array}$ \\
\hline
\end{tabular}




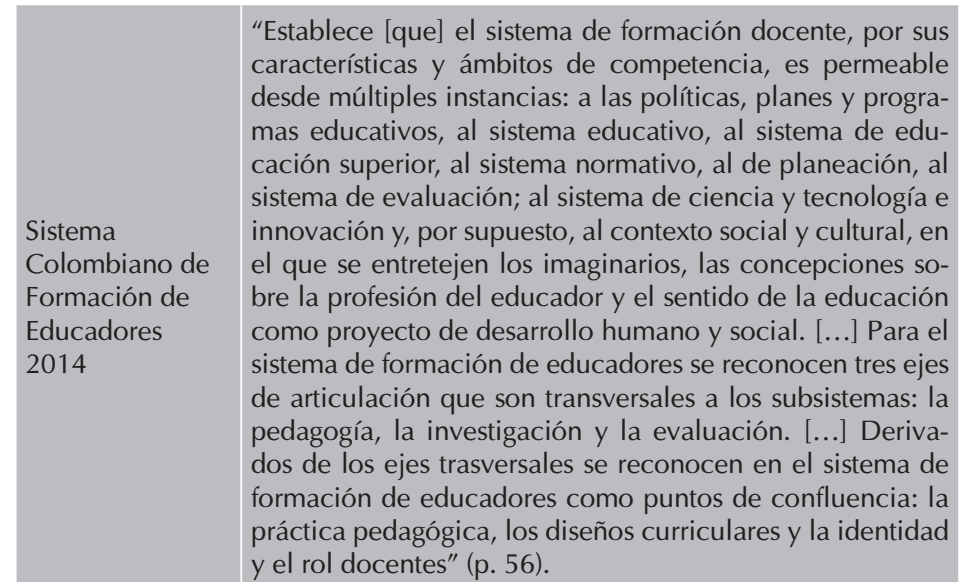

Fuente: elaboración propia con base en documentos de normativa colombiana.

posicionaron como nociones centrales en los discursos acerca de la formación profesional de los maestros, ahora cobijados bajo el nombre de "profesionalización docente". En ese sentido, es necesario aclarar que la forma como ellas aparecen se caracteriza por dos elementos claves: el primero, la amplia cobertura tanto de niveles de formación como de instituciones y procesos encargados de preparar a los docentes; el segundo, el soporte conceptual marcado por tres maneras de entender la relación entre investigación, innovación y formación docente.

El primer elemento se refiere a la necesidad de reconocer que el enunciado de la investigación y la innovación no solo apareció en los escenarios de formación profesional universitaria. Cuando se legisló sobre la formación de maestros ofrecida por las escuelas normales, en perspectiva de adelantar los procesos de reestructuración de estas instituciones, la formación por ellas ofrecida se definió como de carácter inicial y complementario y, en los dos casos, la investigación de forma directa y la innovación de modo indirecto ocuparon un lugar privilegiado, tanto así que se constituyeron en referente de calidad para su acreditación (Marín-Díaz, 2003).

En esta perspectiva, las premisas consagradas en disposiciones normativas, aquellas expresiones de las políticas públicas, fueron la superficie de emergencia de un enunciado de profesionalización docente de los maestros; enunciado que no solo desdibujó la tarea del maestro y su foco de acción, sino que orientó los procesos de su formación hacia prácticas de investigación e innovación sin las cuales las instituciones formadoras de maestros no podían adelantar su tarea formativa y mucho menos alcanzar con éxito la certificación y acreditación de calidad de sus programas. 
De este modo, la formación de maestros en el país se concentró en adelantar procesos de acompañamiento y desarrollo de investigación garantizando que, como investigadores, los maestros fueran partícipes de la producción de un saber capaz de transformar la práctica escolar, esto último sustentado en una necesidad de cambio que aparece como imperativo de muchos relatos sobre la actualidad.

En otras palabras, la investigación y la innovación aparecen vinculadas tanto con la formación como con la producción de saber pedagógico y es por esto que los procesos de profesionalización docente requieren la participación de los estudiantes en procesos investigativos que les permitan por una parte adquirir herramientas para la organización o participación en equipos de investigación y, por otra, "producir resultados y avances en los procesos investigativos, que contribuyan al mejoramiento y la innovación en prácticas y métodos pedagógicos" (Marín-Díaz, 2003, p. 46).

El segundo elemento corresponde a la procedencia y el soporte de los discursos sobre la investigación y la innovación orientadas a la formación docente y que circulan en los documentos de política educativa y en las reflexiones de los investigadores de este campo del saber. Según señalan Aguerredondo y Pogré (2001), los discursos que soportan las perspectivas investigativas en la formación docente podrían agruparse en tres categorías:

... una perspectiva que concibe a la formación inicial como una introducción a los conceptos básicos teóricos relativos a la profesión docente y que entiende que el desarrollo de las competencias profesionales es un desafío a ser enfrentado luego, durante el ejercicio de la profesión; una perspectiva técnica que concibe a la enseñanza como una ciencia aplicada y al docente como un técnico; y una tercera perspectiva que concibe la enseñanza como una actividad crítica y al docente como un profesional autónomo que investiga, reflexionando sobre la práctica, desde la formación inicial (p. 12).

Esas perspectivas se entrecruzan y producen diversas formas de pensar y proceder con relación a la formación de los maestros (ahora pensados como docentes) en investigación e innovación. Una lectura de estas perspectivas en términos de la aproximación que hacen los maestros en ejercicio y en formación a procesos de investigación e innovación como aprendizajes 
básicos para su profesionalización es la que se representa en la figura 1. Esas formas de aproximación (A1, A2 y A3), y sus estrategias y modos de articulación con los demás elementos del proceso formativo se han combinado según las condiciones particulares de cada programa, los formadores encargados de estos procesos, las circunstancias locales y las disposiciones de los maestros en formación, entre otros asuntos.

Figura 1. Tres aproximaciones a la formación de maestros.

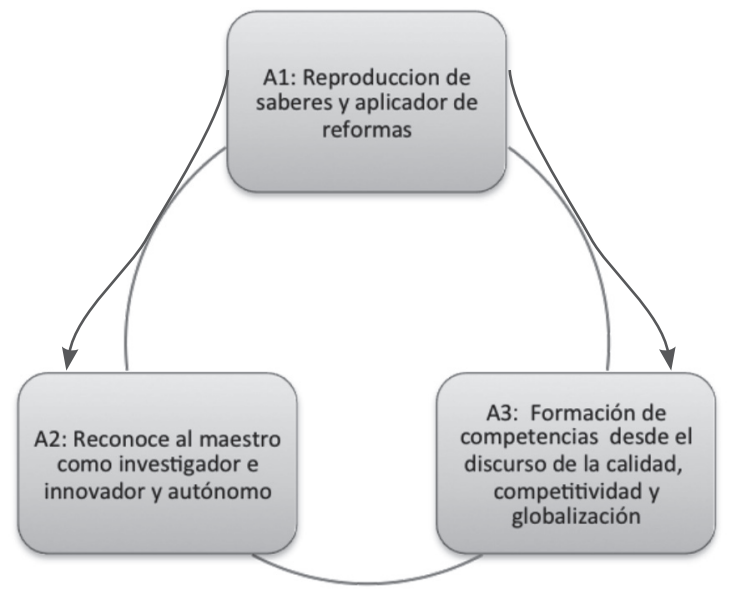

Fuente: elaboración propia.

La primera aproximación (A1) se inscribe en un modelo técnico cuyo núcleo central es la transmisión y la reproducción de saberes en la escuela. En ella, el docente (reproductor de saberes producidos por otros) se encarga fundamentalmente de aplicar las reformas creadas por otros para transformar las prácticas escolares.

La segunda aproximación (A2) se inscribe en un modelo práctico-reflexivo cuyo eje central es la construcción del saber. En ella, el docente es un intelectual de la educación y, en esa medida, se encarga de sistematizar y transformar su propia práctica siendo el factor fundamental de la innovación. Esta es una aproximación que se articula con los aportes recientes de las corrientes didácticas.

La tercera aproximación (A3), tal vez la de más reconocimiento hoy, tiene como núcleo central los discursos sobre la calidad de la educación y la emergencia de las sociedades de la información y el conocimiento. En ella el docente es el sujeto de las competencias, capaz de ejercicios investigativos e innovadores, un sujeto del hacer que responde a las demandas de su tiempo, en particular aquellas de la acreditación, la calidad y la difusión de la innovación, esta última asociada a los usos y apropiaciones de las nuevas tecnologías de la información y la comunicación. 
La aproximación a la investigación y la innovación en los procesos de formación docente no ocurre de un único modo; de hecho, aunque en algunos momentos y programas se ha privilegiado una u otra forma, es posible encontrar que, en la mayoría de casos, la manera como los maestros entran en contacto con ellas resulta de posiciones personales y comprensiones de quienes orientan su formación en un momento determinado, además que ellas entrecruzan diferentes maneras de pensar y entender tales procesos. De modo general se afirma que:

La investigación contribuye a un ejercicio reflexivo, sistemático, crítico, riguroso e innovador que ayuda a que el docente haga mejor su tarea. Posibilita la indagación, sistematización y reconstrucción de su saber pedagógico. La relación investigación-saber pedagógico contribuye al aprendizaje del docente y a su desarrollo profesional, ya que permite avanzar en el conocimiento de enfoques, escuelas, paradigmas, teorías, modelos, metodologías y didácticas que orientan las prácticas pedagógicas cotidianas de los docentes. De igual forma, apoyan los procesos de reflexión sistemática que necesitan desarrollar los maestros para poder identificar logros y dificultades asociadas a la compleja tarea de enseñar y aprender. [...] La investigación que realiza el docente contribuye a la búsqueda del sentido de la acción educativa, inscrita en un contexto institucional y enmarcada en unos lineamientos de política que la orientan. En este orden de ideas, la investigación sobre la práctica docente es un medio para generar y poner a prueba nuevas maneras de enseñar y aprender conducentes a mejorar los estándares de calidad, a dinamizar las instituciones educativas y sus procesos y a lograr que la educación contribuya a la construcción de nación (Camargo, 2005, p. 112).

El aprendizaje docente cuenta con un terreno propicio cuando el maestro participa en propuestas innovadoras. Este aprender mientras se enseña ha sido documentado por Navarro y Verdiso (2000, citados por Terigi, 2006b) a propósito de Programas de Aceleración en el Brasil, en las aulas alternativas en El Salvador y en el Programa de Mejoramiento de la Calidad de la Educación en 
Chile. Como rasgos generales, en estos programas innovadores existen materiales fuertemente estructurados que buscan fortalecer aspectos técnicos del funcionamiento de la enseñanza en estas nuevas condiciones didácticas (Calvo, pp. 153-155).

En ese sentido, se observa en los diseños curriculares organizados para la formación de los maestros (en los modos en que se disponen las prácticas pedagógicas, en la definición de los lugares de esa práctica, en la posición que la investigación ocupa y su relación tanto con la práctica pedagógica como con la innovación, etc.) posturas que con poco o mucho sustento teórico entrecruzan y tejen diferentes perspectivas desde las cuales se aborda la pregunta por la investigación y la innovación.

Esas múltiples formas como en las tres últimas décadas la investigación y la innovación se incorporaron en los procesos de formación de los maestros, asociadas a las políticas de profesionalización docente, fueron el objeto de un estudio adelantado por un equipo de investigación de la Pontificia Universidad Javeriana. En el estudio, que soporta el presente artículo, se buscaba indagar por los objetivos y las estrategias empleados por diferentes programas para formar en investigación e innovación en cuatro campos de conocimiento: Ciencias Sociales, Artes y Humanidades, Ciencias Naturales y Matemáticas, y Educación y Pedagogía. En las siguientes secciones se presentan la metodología usada en el estudio, así como los principales resultados y conclusiones que de él se derivaron; algunas de las reflexiones ya se consideraron en la introducción del artículo.

\section{Sobre la metodología del estudio}

En el estudio titulado Formación en investigación e innovación en programas de licenciatura en los campos de Ciencias Sociales, Artes y Humanidades, Ciencias Naturales y Educación y Pedagogía, que sirve de soporte para este artículo, se indagó por la relación entre investigación e innovación pedagógica a la luz de las preocupaciones por la formación inicial de maestros; esto porque en dicha relación, al parecer, se ponen en juego aspectos de gran relevancia en la formación de maestros, marcados por una orientación de profesionalización docente de carácter técnico-científico.

El diseño metodológico comprendió la recolección y análisis de tres tipos de fuentes:

a. Las propuestas curriculares de dieciséis (16) programas de Licenciatura pertenecientes a universidades de los sectores público y privado que ofrecieran cobertura geográfica de ocho (8) ciudades del país; agrupadas en 
cuatro campos del saber: cinco (5) del área de Educación y Pedagogía, tres (3) del área de Ciencias Sociales, cuatro (4) de las áreas de Educación Artística y Humanidades, y cuatro (4) de las áreas de Ciencias Naturales y Matemáticas.

b. Una encuesta elaborada con la técnica Likert (método de evaluaciones sumarias) que contenía cuatro (4) puntos y se aplicó a 624 estudiantes y 110 docentes pertenecientes a los dieciséis (16) programas de Licenciatura escogidos para el estudio. La encuesta indagó acerca de los objetivos de formación en diecisiete (17) ítems, sobre las estrategias de formación en quince (15) ítems, sobre los espacios de formación en once (11) ítems y algunas preguntas de identificación.

c. Dos grupos focales en cada uno de los programas seleccionados. En total se establecieron dieciséis (16) grupos focales con estudiantes y dieciséis (16) con docentes. Los estudiantes participantes debían ser miembros de semilleros de investigación preferiblemente, o estar vinculados a un proyecto de la Facultad, del programa, o de una línea de investigación. Por su parte, los maestros debían tener un tiempo de vinculación al programa superior a dos (2) años y conocer el funcionamiento del mismo, ya sea porque formaban parte del Comité de carrera o porque desempeñaban funciones de coordinación de espacios académicos.

En general, en el estudio se trabajó con datos cualitativos provenientes de las tres fuentes indagadas y documentadas: los programas curriculares y las transcripciones de grupos focales se analizaron a través de matrices y usando la codificación abierta con el programa Atlas.ti, 7.0. Para el análisis de las encuestas se utilizó un procesamiento de estadística descriptiva.

Los datos obtenidos por medio de la lectura de las propuestas curriculares de los programas seleccionados se usaron como insumos para realizar un análisis documental en dos etapas: una de recopilación de documentos que brindaran información precisa respecto de las preguntas y objetivos del estudio, y la segunda, de descripción-interpretación, que brindara la posibilidad de construir un nuevo documento como aporte de conocimiento acerca del significado de la investigación y la innovación, sus objetivos y estrategias en el contexto de la formación 
de maestros, de acuerdo con el ideal de formación declarado en los planteamientos curriculares (propósitos, perfil, estrategias de formación enunciadas en los documentos).

La información obtenida mediante la encuesta se usó de manera indicativa, dado que la muestra se tomó por participación voluntaria. Con ella se buscaba reconocer un conjunto de percepciones que estudiantes y profesores tenían acerca de los objetivos y las estrategias de formación. Los estudiantes encuestados debían cumplir un requisito: haber cursado más de la mitad de las asignaturas o créditos del programa; por su parte, los maestros encuestados debían ser responsables de asignaturas del componente de investigación y/o de innovación y participar en los espacios académicos asociados con estos componentes.

Finalmente, la información recuperada a través de los grupos focales permitió identificar los objetivos y las estrategias utilizados en el programa de acuerdo con las experiencias formativas vividas por estudiantes $y$ maestros involucrados en espacios académicos del programa. Se orientaron a registrar lo experiencial para trascender lo declarado en los documentos, en los cuales se reflejaban particularmente los requisitos oficiales demandados a los programas. A partir de las respuestas, las opiniones y los comentarios ofrecidos por ellos se realizó un balance descriptivo por medio del cual se caracterizaron los procesos de formación en investigación e innovación implementados en los programas. Así se pudieron percibir algunas de las tendencias y estilos que aparecen como orientadores de dicha formación y las tensiones emergentes en esos ámbitos de formación.

\section{Sobre las concepciones de investigación e innovación: algunos resultados}

En los párrafos siguientes se enuncian tres resultados obtenidos al realizar la lectura de la información cualitativa obtenida en el estudio, sin embargo es preciso aclarar que ellos no fueron los únicos y que para efectos informativos se escogieron por constituir un marco amplio en el que se localizan otros resultados específicos para cada una de las áreas estudiadas. El primer resultado se refiere a los principales propósitos que orientan la formación en investigación, los cuales no son necesariamente excluyentes entre sí; el segundo resultado da cuenta de las concepciones acerca de la innovación que transitan en la formulación de los programas y que aparecen como respuestas a necesidades formativas específicas; finalmente, el tercer resultado se refiere a la articulación que se propone y argumenta entre la investigación y la innovación como elementos de la práctica profesional docente.

Sobre el primer resultado, se puede decir que son cinco los objetivos que aparecen como característicos de las formas y los argumentos usados para explicar las prácticas de 
formación en investigación dentro de los programas: a) dominio de elementos básicos de la investigación; b) resignificación de la profesión docente; c) transformación de la práctica; d) construcción de los conocimientos sobre sujetos educativos; y e) ampliación de la intencionalidad de la práctica: de reproductora a productora de conocimiento. En la figura 2 se presentan de forma esquemática estos propósitos y se intenta mostrar que ellos no se encuentran desligados los unos de los otros; es más, en la mayoría de los casos en un mismo programa dos o tres de estos elementos se usan como argumentos para promover la formación en investigación de los profesionales docentes.

Figura 2. Objetivos de formación en investigación en programas de licenciatura.

A

Dominio de elementos básicos de la investigación

E

Ampliación de la intencionalidad de la práctica: de reproductor a productor de conocimiento

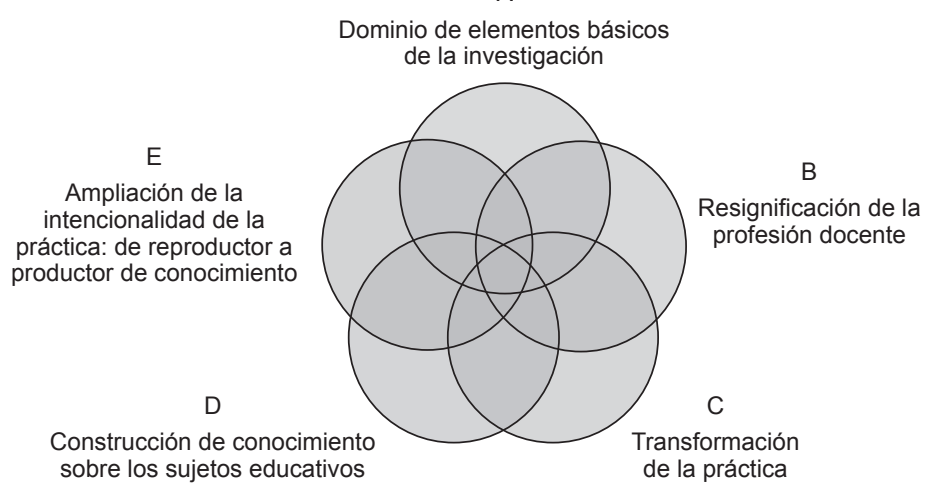

Fuente: elaboración propia.

El objetivo A ubica la investigación como un contenido curricular sobre el cual el licenciado debe tener conocimientos básicos, con ello se centra en la existencia de cursos de investigación que proporcionan conceptos, métodos y técnicas requeridas para formular un proyecto de investigación. Los objetivos B y C atribuyen a la investigación un papel asociado a la tarea de resignificar la profesión docente, es decir que la inclusión de la investigación como capacidad del maestro para investigar le otorga rasgos que lo hacen profesional docente, como, por ejemplo, una actitud de indagación, una postura crítica y un sentido de la transformación de su propia práctica. Vale la pena señalar que la noción maestro-investigador, asumida como función inherente a la profesionalidad docente, fue una apuesta de los maestros que formaron parte del Movimiento Pedagógico y de algunos intelectuales de la educación. 
Los objetivos D y E corresponden a un posicionamiento del pensamiento crítico-transformador de la educación que considera al docente como productor de conocimiento y no solo como reproductor de saberes elaborados por otros. En esta tendencia se ubican los objetivos de formación comprometidos principalmente con pedagogías críticas.

Sobre el segundo resultado, acerca de las concepciones sobre la innovación que aparecen en los programas, es preciso señalar que son cinco las necesidades que se evidencian en los argumentos expuestos para justificar la formación en innovación: a) la necesidad de cambio y mejoramiento de un campo determinado de la vida social; b) la necesidad de acciones pertinentes y contextualizadas; c) la necesidad de tomar distancia frente a las prácticas tradicionales; d) la importancia de hacer de la innovación un elemento constitutivo de la práctica pedagógica; y e) la producción de espacios de autonomía y decisión para el maestro abriendo escenarios de acción política para el maestro.

En la figura 3 se presentan de manera esquemática las principales necesidades que soportan la urgencia de formar a los maestros en innovación. A pesar de la diversidad de comprensiones asociadas a esta idea de innovación, es posible reconocer tres concepciones: la primera, vinculada a las necesidades $A$ y $B$, propone la innovación como el cambio y mejoramiento que tienen lugar en distintos campos de la actividad social; generalmente se trata de mejorar prácticas y procesos existentes y producir un valor agregado. Esta se percibe, además, como una acción pertinente por cuanto está contextualizada y da respuesta a problemáticas concretas.

Figura 3. Necesidades que subyacen a las estrategias en la formación para la innovación en programas de licenciatura.

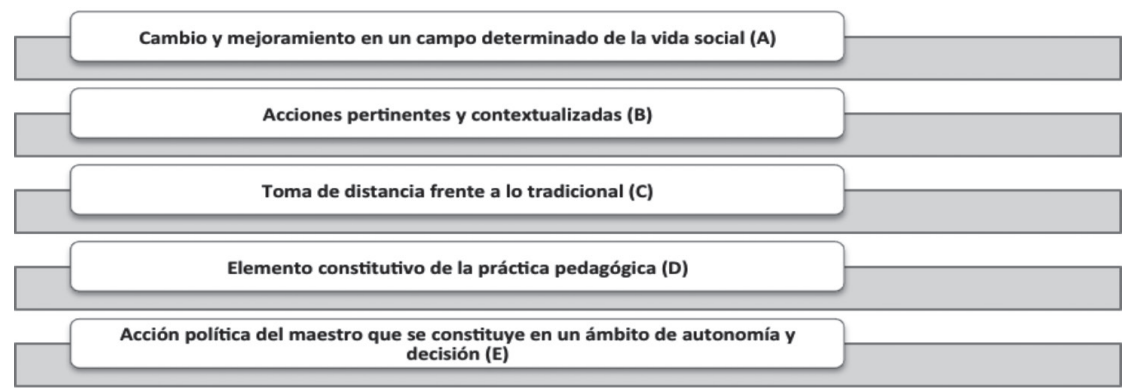

Fuente: elaboración propia.

La segunda concepción, vinculada a la necesidad $\mathrm{C}$, define la innovación en contraste con lo tradicional en la medida que plantea alguna ruptura respecto de lo convencional o lo rutinario. En este 
sentido, lo tradicional aparece asociado con formatos rígidos de la enseñanza y con formatos jerárquicos y homogenizantes de la vida escolar.

La tercera concepción se propone como respuesta a las necesidades $\mathrm{D}$ y $\mathrm{E}$, se formula como una perspectiva complementaria de las anteriores y, en ese sentido, sería la dimensión ontológica, política y ética de la innovación. Dos son los sentidos que adquiere la innovación en este caso: por un lado, se concibe como un espacio de autonomía del maestro, escenario de empoderamiento en el cual puede y debe tomar las decisiones sobre la enseñanza; por otro lado, se propone como un rasgo constitutivo de las prácticas pedagógicas y no una externalidad impuesta; en ese sentido se parte del supuesto de que "ninguna situación pedagógica, incluso la más tradicional, puede pervivir y sostenerse en el tiempo sin cambiar algo" (Aguilar, 2010, p. 3).

Sobre el tercer resultado, referido a la articulación que se propone y argumenta entre la investigación y la innovación como elementos clave de la formación profesional docente, es preciso señalar dos puntos: el primero sobre la relación que se formula entre las dos prácticas, el segundo sobre los principales escenarios en donde ocurre esa formación.

Primer punto. Aunque en las formulaciones de los programas, en las entrevistas y en los grupos focales aparece la necesidad de diferenciar la investigación de la innovación, las relaciones que se establecen entre estas dos prácticas parecen diluir sus diferencias y con ellas sus propósitos y ámbitos particulares. En ese sentido, es posible encontrar que a veces aparecen señaladas como una misma acción que cumple un mismo propósito; otras veces como una cadena continua que lleva de la investigación a la innovación y viceversa.

Los siguientes ejemplos ilustran mejor la forma como se presentan estas relaciones, de acuerdo con testimonios de estudiantes:

\section{P 7: Codes: [1.1 Qué es la investigación]}

Yo creo que la investigación nace de la reflexión de la práctica pedagógica, en el momento en que uno se encuentra en una realidad y en un contexto y reflexiona sobre eso, directamente lo lleva a que investigue y esa investigación directamente lo lleva a que innove para que pueda funcionar todo. Entonces cuando uno está comprometido y ama lo que hace, reflexiona, se está cuestionando y se está preguntando todo el tiempo sobre lo que está pasando en esa realidad. 


\begin{abstract}
P 5: Codes: [1.2 Relación con la innovación]

...el espacio de prácticas es el único lugar apropiado para pensar ese tema porque es precisamente donde se está en contacto con el campo, con el lugar donde uno va a desarrollar su práctica y en este caso, entonces es un espacio que a mí me parece adecuado y casi perfecto para tratar ese punto del vínculo entre la práctica, la innovación y la investigación, cómo se articulan esas tres cosas para lograr un proceso educativo o formativo, que tenga esos tres aspectos que le sirven a uno como maestro y que le van a aportar principalmente a la población con la que uno está trabajando.
\end{abstract}

Al respecto, Moreno (2000) señala que tal vez sea por el ámbito de desarrollo, el escenario educativo, los procesos de innovación se identifican con la investigación educativa, pues los dos se formulan como espacios para la transformación de las prácticas escolares y no se vinculan necesariamente con la producción de conocimiento.

Segundo punto. Al indagar sobre los espacios académicos asociados con la formación en investigación e innovación, es interesante observar que las asignaturas de metodología de la investigación, las prácticas pedagógicas, los seminarios de énfasis y electivos y el trabajo de grado aparecen como los espacios institucionalizados que cuentan con una tradición para garantizar esta formación. Se trata de escenarios que han alcanzado mayor despliegue y organización al ser mostrados como lugares formales donde se aprende a investigar e innovar $y$, por eso mismo, como espacios donde se puede evidenciar que la institución cumple con el también requisito de calidad exigido para la acreditación de sus programas de formación. En la tabla 2 se resumen las valoraciones que de estos espacios hacen los estudiantes, cuando los interrogan acerca de la formación que reciben.

Es interesante señalar que los semilleros de investigación son, sino la principal, una de las principales estrategias de formación en investigación referida, descrita y valorada por el grupo de docentes y estudiantes participantes en el estudio. Cuatro características sobresalen en las descripciones sobre la forma como opera esta estrategia: la primera, desinstitucionalización e informalidad de los semilleros de investigación, significa que el semillero aparece como un escenario de formación en investigación por fuera del plan de estudios oficial y se constituye en el espacio opcional para aprender a investigar con otros, ya sean personas con experiencia en investigación -generalmente profesores con líneas de investigación o proyectos a su cargo- u otros estudiantes que están interesados en participar de estos procesos, sea por la necesidad de sacar adelante sus proyectos de grado, por curiosidad 
Tabla 2. Resumen de las valoraciones de los estudiantes en la escala sobre espacios académicos asociados a la investigación e innovación.

\begin{tabular}{|c|c|c|c|}
\hline & Bajo & Medio & Alto \\
\hline Prácticas pedagógicas en instituciones educativas & $7.7 \%$ & $27.4 \%$ & $64.9 \%$ \\
\hline $\begin{array}{l}\text { Trabajo de grado en una línea de investigación del } \\
\text { programa o de la institución }\end{array}$ & $10.2 \%$ & $31.1 \%$ & $58.7 \%$ \\
\hline Asignaturas de metodología de investigación cursadas & $15.4 \%$ & $40.0 \%$ & $44.6 \%$ \\
\hline Semillero de investigación en el que participa & $26.8 \%$ & $30.7 \%$ & $42.5 \%$ \\
\hline $\begin{array}{l}\text { Seminarios en los que se combinan } 2 \text { o más campos de } \\
\text { conocimineto para obtener miradas novedosas de la educación }\end{array}$ & $23.9 \%$ & $39.7 \%$ & $36.4 \%$ \\
\hline $\begin{array}{l}\text { Grupo o proyecto de acompañamiento y asesoramiento a } \\
\text { escuelas }\end{array}$ & $28.6 \%$ & $38.1 \%$ & $33.2 \%$ \\
\hline Seminarios de énfasis y electivos & $21.8 \%$ & $47.3 \%$ & $30.9 \%$ \\
\hline $\begin{array}{l}\text { Trabajo de auxiliar de investigación en un proyecto de la } \\
\text { facultad }\end{array}$ & $33.6 \%$ & $40.9 \%$ & $25.5 \%$ \\
\hline $\begin{array}{l}\text { Grupo de trabajo conjunto con otros profesionales (por ejemplo } \\
\text { psicólogos) en la solución a problemas educativos }\end{array}$ & $39.8 \%$ & $35.4 \%$ & $24.8 \%$ \\
\hline $\begin{array}{l}\text { Grupo de actividades con maestros en ejercicio para solucionar } \\
\text { problemas de contextos concretos }\end{array}$ & $30.6 \%$ & $45.0 \%$ & $24.4 \%$ \\
\hline $\begin{array}{l}\text { Grupo, red o comunidad virtual para la discusión de } \\
\text { problemáticas educativas }\end{array}$ & $39.8 \%$ & $39.2 \%$ & $21.0 \%$ \\
\hline
\end{tabular}

o por el interés de adquirir herramientas para realizar otras investigaciones.

La segunda característica que agrupa las distintas referencias que se hacen a los semilleros como estrategias de formación en investigación es la jerarquización interna de la organización del semillero. Tal jerarquización aparece referida, en lo fundamental, al tiempo de permanencia de los estudiantes en el semillero. En unos casos, los estudiantes ingresan al semillero desde el inicio de la licenciatura; esa vinculación generalmente se consigue cuando ellos llegan a trabajar con un profesor a un proyecto que él dirige y que les sirve para aprender a investigar. En el caso de una vinculación tardía, que ocurre en los últimos semestres, el principal motivo que lleva a los estudiantes a ingresar al semillero es realizar el proyecto de grado. Tales diferencias en la vinculación y permanencia dentro del semillero parecen ser el motivo para que haya cierta "jerarquización" en el interior del mismo.

La tercera característica que agrupa las distintas referencias que se hacen a los semilleros como estrategias de formación en investigación es la tensión entre el enfoque disciplinar y el enfoque pedagógico de las investigaciones que se desarrollan. Las respuestas ofrecidas por docentes y estudiantes señalan una permanente pregunta por la naturaleza disciplinar o pedagógica de los proyectos que los congregan en estos grupos. En la mayoría de los casos, se aclara que el programa de licenciatura ofrece formación en investigación tanto en el campo disciplinar (o el saber específico) como en el campo pedagógico (y/o didáctico) 
y que la vinculación al semillero depende de la decisión del estudiante, según sus intereses mayores por uno u otro tipo de formación.

La cuarta y última característica, que agrupa las distintas referencias que se hacen a los semilleros como estrategias de formación en investigación es su articulación a otras estrategias de formación en investigación: articulación con los trabajos de grado, articulación con los grupos de investigación y articulación con la práctica pedagógica. La primera de esas vinculaciones, la de los semilleros con los trabajos de grado, se puede percibir en varias de las citas usadas anteriormente $y$ en las cuales los semilleros aparecen como el espacio de formación en investigación y para la producción de los trabajos de grado. La segunda de esas vinculaciones es entre semilleros y grupos de investigación.

A continuación y como una de las principales conclusiones del estudio se describe el que puede ser entendido como un campo de tensiones que surge del análisis adelantado y que permite arriesgar una hipótesis: parece estar fortaleciéndose la idea de un maestro-investigador, no tanto porque asuma una posición crítica y analítica frente a su quehacer como porque está sumergiéndose paulatinamente en los mecanismos de producción científica de las universidades y del Sistema Nacional de Ciencia, Tecnología e Innovación del país (Cabra-Torres, Herrera, Gaitán, Castañeda-Peña, Garzón, Marín-Díaz,
García-Cepero, Barrios-Martínez y Jiménez-Muñoz, 2013).

\section{Conclusiones:} la formación en investigación e innovación como campo de tensiones

La pregunta general que orientó el estudio se ubicó en la perspectiva de aprender de las experiencias de los estudiantes y de maestros que contribuyen a la formación de los futuros licenciados. El conjunto de cuestiones problemáticas manifestadas por profesores y estudiantes de los programas analizados se relacionan con tres aspectos centrales: aquellos que tienen que ver con algunas nociones subyacentes a la formación (práctica pedagógica, cambio, innovación, etc.); las estrategias utilizadas para formar al maestro como investigador e innovador, y la dimensión curricular de la formación. Las principales tensiones identificadas pueden resumirse en los siguientes aspectos, que interrogan su campo de profesionalización.

- En relación con la noción de práctica pedagógica lo que se observa es ausencia de claridad epistemológica: al reducir la práctica pedagógica a "lo práctico", se produce un empobrecimiento del saber pedagógico debido al aplicacionismo en que se ubica (aplicar teorías, métodos, estrategias, soluciones). 
Adicionalmente, se infiere una relación poco precisa entre esta y la innovación. Se supone, en términos generales, que los cambios generacionales implican una serie de relaciones que obligan a replantear la práctica pedagógica, y se carece de una visión comprensiva de lo que significaría formar al maestro para innovar.

Con respecto a la relación entre los formadores de formadores y los maestros en ejercicio, se presenta una serie de desarticulaciones que evidencian el escaso contacto de los profesores, que están al frente de los programas de licenciatura, y las escuelas, y en general con los espacios educativos. Esto se manifiesta en un desconocimiento de las dinámicas y problemáticas reales de las instituciones escolares, y en la imposición de teorías explicativas de los fenómenos educativos, por encima de la diversidad y complejidad institucional y existencial de las prácticas educativas.

Adicionalmente, se alude en términos de ser una tensión presente y persistente el hecho de que la interacción generacional entre los maestros en ejercicio y los estudiantes de las licenciaturas son relaciones de interaprendizaje poco reflexionadas y potencializadas, en las cuales priman las asimetrías y jerarquías.

- De otra parte, las posibles interrelaciones entre investigación e innovación son relaciones difusas y lineales, sin claridad teórica. Se observa, además, que las nociones de cambio educativo y de transformación son poco explícitas, se prescriben sin un contenido clarificador.

- Las visiones sobre la innovación que prevalecen en las prácticas de formación de los futuros maestros resultan ser un campo abierto a la indagación. La tensión que prevalece es la insuficiente diferenciación entre las actividades de investigación y las de innovación, y la visión de la innovación como resultado natural de la investigación que deja de interpelar el origen y propósito de la innovación en sí misma volviéndola una acción rutinaria.

Prevalecen visiones de la innovación como: "usar tecnologías es innovación"; "asumir campos de acción con diferentes poblaciones es innovación". A la vez, 
se concibe la innovación que promueven el Estado y la reforma educativa como "coyuntural, instrumental y modernizante sin grandes alcances o impactos".

A lo anterior se añaden las escasas oportunidades de innovar en algunas instituciones educativas donde todo está establecido y no hay libertad para inaugurar alternativas a lo existente, situaciones críticas de la formación del licenciado en las que la innovación se queda en el papel.

- Por su parte, la relación entre investigación y campos disciplinares (Ciencias $\mathrm{Na}$ turales, Ciencias Sociales, Artes y Humanidades, Educación y Pedagogía) suele reducir la práctica a lo disciplinar sin articularla con el saber pedagógico, con el agravante de que este queda minusvalorado respecto del disciplinar, lo cual produce un efecto perverso y naturalizado con respecto a la mayor deslegitimación y menor validación del saber del maestro.

La investigación que se proyecta en los espacios académicos no siempre está orientada a la producción de conocimiento, en cambio se encuentra más asociada a la reflexión, al mejoramiento de la práctica educativa, o a relaciones pertinentes con los contextos. Al convertir la investigación en un requisito o mecanismo de graduación, se produce un empobrecimiento de la cultura académica y científica de la formación de los futuros maestros, debido a la normalización de estándares que se plantea desde las reformas basadas en la profesionalización docente.

A pesar de las bondades y beneficios que para la formación del maestro colombiano puedan traer la investigación y la innovación como orientaciones de la política de profesionalización docente, conviene indagar por los límites de la investigación sobre la práctica, en últimas, es decir, hasta dónde debe llegar, y cuál es la relación entre un maestro en formación cuando aprende su oficio de enseñante, y cuando se convierte en investigador o innovador de su propia práctica docente o de otras experiencias externas. Esto es, preguntarse si los procesos investigativos sobre la práctica limitan las experiencias pedagógicas desde las que el maestro construye su identidad profesional. Así, estarían en discusión las visiones que plantean al maestro como 
investigador y productor de conocimiento como exigencia de la profesionalización docente, y no necesariamente como un modo de reconocerse como portador y productor de saber pedagógico, y como un modo de hacer uso de su autonomía sin limitarse a las exigencias de agentes externos.

... [el] maestro que comprende que su tarea no se reduce a pensar en el cumplimiento de los estándares de calidad basados en pruebas estandarizadas, en tanto encuentra que la realidad escolar desborda este referente y por tanto encuentra múltiples posibilidades para asumirse maestro e investigador de la pedagogía (Suárez y Ortiz, 2010, p. 333).

Finalmente, se hace indispensable evaluar el impacto de las estrategias empleadas en la formación para la investigación y la innovación de acuerdo con lo que se espera a nivel de pregrado en los procesos de profesionalización docente. Se propone un desplazamiento de los modelos de evaluación basados en resultados de competencias terminales de los futuros licenciados, a la indagación de la formación centrada en los procesos de mediación, desarrollo de capacidades y en el sujeto de saber que se está formando en dichas instancias.

\section{Referencias bibliográficas}

Aguerrondo, I. y Pogré, P. (2000). Las instituciones de formación docente como centros de innovación pedagógica. Buenos Aires: Editorial Troquel.

Aguilar, J. F. (2010). Innovaciones educativas y alternativas pedagógicas: una distinción necesaria (aunque problemática). Documento de la conferencia inaugural del Tercer Encuentro Interinstitucional de Prácticas en la Formación de Docentes para la Infancia. Las prácticas formativas en diversidad de escenarios y sus implicaciones ético-políticas, socioculturales, pedagógicas y didácticas.

Álvarez Gallego, A. (2011). Los maestros investigadores. De posturas, supuestos y campos. Actualidades Pedagógicas, 57, 15-32.

Cabra-Torres, F.; Marín-Díaz, D. L. et ál. (2013). La investigación e innovación en la formación inicial de docentes. Aportes para la reflexión y el debate. Bogotá: Javegraf. 
Calvo, G. (2010). Innovación e investigación sobre aprendizaje docente y desarrollo profesional. En: Vélaz de Medrano, C. y Vaillant, D. Aprendizaje y desarrollo profesional docente (pp. 148-157). Madrid: OEl, Fundación Santillana.

Galvin, I. (2006) La profesión docente en España, retos de futuro. Una perspectiva sindical. En Tenti Fanfani, E. (comp.), El oficio de docente. Vocación, trabajo y profesión en el siglo XXI, (pp. 303-327). Avellaneda: Siglo XXI.

Marín-Díaz, D. (2003). Investigación y formación de docentes en la escuela normal superior. Análisis y perspectivas. Pedagogía y Saberes, 19, 43-52.

Martínez Boom, A. (2000). Malestar docente y profesionalización en América Latina. Revista Española de Educación Comparada, 6, 87-112.

Martínez Boom, A. (2004). De la escuela expansiva a la escuela competitiva. Dos modos de modernización en América Latina. Bogotá-Barcelona: Convenio Andrés Bello, Anthropos.

Martínez Boom, A. (2010a). La dilución del maestro. Reflexiones. Palabra Maestra, 25, 5.

Martínez Boom, A. (2010b) Profesionalización: ¿Oficio, profesión, función? En: Revista Educación y Cultura, 88, 27-32.

Orealc/Unesco (2012). Antecedentes y criterios para la elaboración de políticas docentes en América Latina y el Caribe.
Santiago de Chile: Unesco. Disponible en: http://www.orealc. cl/educacionpost2015/wp-content/blogs.dir/19/files_mf/antec edentesycriteriosparapol\%C3\% ADticaspublicasparadocentesfinal.pdf

Sancho, G. J. y Hernández, F. (2004). ¿Por qué no ha fructificado la propuesta del profesor como investigador? Y algunas propuestas para resistir a un presente nostálgico. Educar, 34, 39-51. Universitat de Barcelona. Disponible en: http://ddd.uab.es/ pub/educar/0211819Xn34p39. pdf

Suárez, J. P., y Ortiz, R. (2010) El maestro como sujeto de saber pedagógico: ¿Es un maestro investigador? (pp. 321-334). En: A. Martínez Boom y A. Álvarez Gallego (Comp.) Figuras contemporáneas del maestro en América Latina. 30 años del Grupo de Historia de la Práctica Pedagógica. Bogotá: Editorial Magisterio.

Vaillant, D. (2002) Formación de formadores. Estado de la práctica. San Marino: Preal.

Vezub, L. (2007) La formación y el desarrollo profesional docente frente a los nuevos desafíos de la escolaridad. Profesorado. Revista de Currículum y Formación del Profesorado 11(1), 1-23.

Zambrano Leal, A. (2012). Formación docente en Colombia. Arqueología de la política pública y del escenario internacional. Educere, 16(54), 11-18. 